\title{
Effect of Oil Content and Emulsifier Type on the Properties and Antioxidant Activity of Sea Buckthorn Oil-in-Water Emulsions
}

\author{
Hongxia Zheng $\left(\mathbb{D},{ }^{1}\right.$ Like Mao $\mathbb{D}^{1,{ }^{1,2}}$ Jingyi Yang, ${ }^{1}$ Chenyu Zhang, ${ }^{1}$ Song Miao, ${ }^{3}$ \\ and Yanxiang Gao ${ }^{1}$ \\ ${ }^{1}$ Beijing Advanced Innovation Center for Food Nutrition and Human Health, Beijing Laboratory for Food Quality and Safety, \\ College of Food Science \& Nutritional Engineering, China Agricultural University, Beijing 100083, China \\ ${ }^{2}$ Xinghua Industrial Research Centre for Food Science and Human Health, China Agricultural University, Xinghua 225700, \\ Jiangsu, China \\ ${ }^{3}$ Teagasc Food Research Centre, Moorepark, Fermoy Co. Cork T12K8AF, Dublin, Ireland
}

Correspondence should be addressed to Like Mao; likemao@cau.edu.cn

Received 7 August 2019; Revised 2 November 2019; Accepted 22 November 2019; Published 13 January 2020

Academic Editor: Antonio Piga

Copyright $\odot 2020$ Hongxia Zheng et al. This is an open access article distributed under the Creative Commons Attribution License, which permits unrestricted use, distribution, and reproduction in any medium, provided the original work is properly cited.

\begin{abstract}
Sea buckthorn oil-in-water emulsions were prepared through high pressure homogenization, and the effects of droplet size, oil content, and emulsifier type on emulsion properties and the overall antioxidant activity of the emulsions were evaluated. Emulsions with different droplet size were obtained by varying homogenization pressure, and higher oil content resulted in bigger droplet size of the emulsions. Among three tested emulsifiers, sodium caseinate and sugar ester were able to form emulsions with much smaller particle size than soy protein isolate. The emulsions with bigger droplets tended to cream in an accelerated centrifugation test. The antioxidant property of the emulsions was expressed as their DPPH radical scavenging activity. The emulsions processed at lower pressure or contained higher oil content had higher DPPH radical scavenging activity. The soy protein isolate-stabilized emulsion presented higher antioxidant activity than sodium caseinate- and sugar ester-stabilized ones. Upon storage, the antioxidant activity of the emulsions was decreased due to the changes in emulsion stability and the degradation of antioxidants. The knowledge obtained in this study would be useful in developing healthy food containing sea buckthorn oil.
\end{abstract}

\section{Introduction}

Sea buckthorn (Hippophae rhamnoides L.) is widely distributed in China, Mongolia, Russia, Northern Europe, and Canada, particularly in dry or sandy areas, e.g., sea coasts, dry semidesert sites, and high mountains. It is a hardy plant, drought- and cold-resistant, and thus, it is usually used for soil, water, and wildlife conservation and antidesertification purposes [1]. Berries of sea buckthorn have long been used in oriental countries for the treatment of gastrointestinal diseases, skin disorders, asthma, hepatitis, rheumatism, and so on. Recent studies have revealed that berries of sea buckthorn, mainly their oil extracts, are rich in bioactive compounds, including monounsaturated fatty acids, vitamins, carotenoids, and phytosterols $[2,3]$. Sea buckthorn oils are thus gaining more popularity in the formulation of healthy foods as sources of bioactive supplements and antioxidants $[4,5]$. On the contrary, it is important to protect sea buckthorn oil from oxidation by environmental stresses (e.g., oxygen, heat, and enzymes).

Oil-in-water emulsions are generally regarded as good delivery systems for functional oils (e.g., fish oils, flax seed oils, and safflower oil) and oil-soluble bioactives (e.g., $\beta$-carotene, $\alpha$-tocopherol, and coenzyme Q10) to modify the dispersant state, to mask off-flavors, and to improve digestion and uptake [6]. When dispersed in the oil droplets of an oil-in-water emulsion, oil-soluble ingredients are isolated from the external environment by the oil-water interface and water phase, and thus may have improved stability [7]. Therefore, properties of the oil droplets and the interfacial films are critical factors determining the stability and functionality of the bioactives within. Emulsions with 
smaller particle size (e.g., nanoemulsions) are usually preferable not only because that these emulsions have better physical stability and possible to make transparent food systems [8], but also that bioactives in smaller particles are easily adsorbed [6]. However, small particles are not favored for the stabilization of bioactives in emulsions upon storage because of the large interfacial area covering the oil-water interface, which allows more exposure of the oil droplets to the environment [9]. Nevertheless, a proper selection of emulsifiers allows the formation of compact interfacial layers to block the attacks from the environment, and thus protecting the bioactives from degradation [10]. Among different emulsifiers, large molecular weight emulsifiers (e.g., protein, polysaccharides) are usually more effective in improving the stability of bioactives because of the thicker interface formed and the possible antioxidant groups available in the molecules [11]. Although the effects of droplet properties and emulsifiers on the stability of many bioactives have been widely reported [7], the effects on the antioxidant properties of the emulsions containing bioactives were less investigated, especially for oil extracts with mixed bioactives. Antioxidant activity of a specific bioactive is largely dependent on its chemical structure and concentration but also related to the properties of the carrier systems. The well-known "polar paradox theory" suggested that lipophilic antioxidants are more effective in oil-wateremulsions than in bulk oils due to their higher affinity for the oil-water interface, where they form protective barriers around the droplets [12]. However, some later studies reported that not all antioxidants followed this theory, and the complexity of the use of antioxidants in food emulsions requires extensive investigations [13]. On the contrary, oil natures (e.g., chain length, saturation degree, and solid lipid content) largely affect emulsion properties (e.g., size, viscosity, and stability), as well as the bioaccessibility of the bioactives incorporated $[14,15]$.

From the literature, the microencapsulation of sea buckthorn oils has been well studied, mostly focusing on the roles of wall materials and storage conditions on the stability of sea buckthorn oils $[16,17]$. The preparation of emulsions is an important step of the microencapsulation process, and properties of emulsions greatly affect the stability and functionality of the microencapsulated products [18]. However, only minor attention has been paid to sea buckthorn oil-in-water emulsions [19]. It was known that oil content and emulsifier type play essential roles in emulsion properties [8], and the objective of the current study was to investigate the effect of emulsion properties, as affected by oil content and emulsifier type, on the antioxidant activity of sea buckthorn oil-in-water emulsions. We selected three commonly used food emulsifiers, i.e., soy protein isolate (SPI), sodium caseinate (SC), and sugar ester (SE) to form the emulsions.

\section{Materials and Methods}

2.1. Materials. Sea buckthorn oil was kindly offered by Beijing Powdery Food Technology Co., Ltd. (Beijing, China). The oil was extracted by supercritical $\mathrm{CO}_{2}$ from dried sea buckthorn berries (with seeds), which were collected in Xinjiang, China. As the supplier stated, the oil contained $35.92 \mathrm{~g} / 100 \mathrm{~g}$ palmitic acid, $34.91 \mathrm{~g} / 100 \mathrm{~g}$ palmitoleic acid, $12.59 \mathrm{~g} / 100 \mathrm{~g}$ linoleic acid, $6.28 \mathrm{~g} / 100 \mathrm{~g}$ oleic acid, $1.08 \mathrm{~g} /$ $100 \mathrm{~g}$ steric acid, and $0.69 \mathrm{~g} / 100 \mathrm{~g} \alpha$-linolenic acid. The oil was centrifuged to remove any precipitates before use. Sodium caseinate (SC) was obtained from Tatua Cooperative Dairy Company Ltd. (Morrinsville, New Zealand). Soy protein isolate (SPI) was purchased from Yuanchuang Biological Technology Co., Ltd. (Hebei, China). Sucrose ester (SE) was the product of Gaotong Food Technology Co., Ltd. (Guangxi, China). Sodium azide and 1,1-diphenyl-2-picrylhydrazyl (DPPH) were bought from Sigma-Aldrich (St. Louis, MO, USA).

2.2. Emulsion Preparation. SC, SPI, or SE $(1 \mathrm{~g} / 100 \mathrm{~g}$ of final emulsion) was dispersed in deionized water and kept overnight to ensure complete hydration. Sodium azide $(0.01 \mathrm{~g} / 100 \mathrm{~g})$ was added as an antimicrobial agent. Sea buckthorn oil $(5,10$ or $20 \mathrm{~g} / 100 \mathrm{~g})$ was mixed with the emulsifier dispersion at $10000 \mathrm{rpm}$ for $10 \mathrm{~min}$ using an Ultra-Turrax high-speed blender (IKA, Germany) to form a coarse emulsion, which was further homogenized using a Niro Soavi Panda high-pressure homogenizer (Parma, Italy) at 20, 40, 60, or $80 \mathrm{MPa}$ for 3 cycles. $\mathrm{pH}$ of the emulsions was adjusted to 7.0 with $0.5 \mathrm{M} \mathrm{HCl}$ or $\mathrm{NaOH}$. The final emulsions were immediately cooled down to room temperature $\left(\sim 20^{\circ} \mathrm{C}\right)$ before analysis. For the storage test, emulsions were transferred into screw-capped brown bottles and stored at $20^{\circ} \mathrm{C}$ for $6 \mathrm{~d}$ and sampled for particle size and antioxidant activity determination every $3 \mathrm{~d}$.

2.3. Measurement of Particle Size and Zeta Potential. Oil droplet size and zeta potential of the emulsions were determined by dynamic light scattering using a Zetasizer Nano-ZS90 (Malvern Instruments, Worcestershire, UK) at a fixed detector angle of on $90^{\circ}$. The equipment measured the changes in the intensity of scattered light due to the Brownian motion, which was then converted to droplet size based on Stokes-Einstein equation. Electrophoretic mobility was measured to calculate zeta potential based on the Henry equation. To minimize multiple scattering effect, emulsions were diluted with deionized water prior to each measurement. Results were described as intensity mean diameter $(\mathrm{nm})$ for droplet size, polydispersity index (PDI) for size distribution, and $\mathrm{mV}$ for $\zeta$-potential.

2.4. Emulsion Stability Analysis. Creaming stability of emulsions was evaluated using a multisample analytical centrifuge (LUMiSizer, LUM GmbH, Berlin, Germany) [20]. Emulsions were transferred to rectangular cells $(2 \times 8 \mathrm{~mm})$ and analyzed by a light beam emitted at near infrared wavelength $(880 \mathrm{~nm})$ which scanned the sample cells over the total length. The charge-coupled device (CCD) line sensor received light transmitted through the sample, which showed a pattern of light flux as a function of the radial position, giving a macroscopic fingerprint of the sample at a 
given time, from which emulsion instability, such as creaming, sedimentation, and droplet aggregation, could be detected. In the current study, the samples were centrifuged at $1500 \mathrm{rpm}(286.8 \times \mathrm{g})$ and $25^{\circ} \mathrm{C}$ with a scanning rate of once every $60 \mathrm{~s}$ for $4.2 \mathrm{~h}$.

2.5. Antioxidant Activity Evaluation. Antioxidant activities of sea buckthorn oil-in-water emulsions were evaluated through a DPPH radical method [21]. Briefly, the DPPH radical was dissolved in methanol to reach the concentration of $1 \times 10-4 \mathrm{~mol} / \mathrm{L}$. For radical scavenging activity (RSA) determination, the solution of DPPH radical was added to each emulsion sample in $1: 1$ ratio. Similarly, the control was prepared by adding the solution of the $\mathrm{DPPH}$ radical to methanol in 1:1 ratio. And finally, the blank solution was prepared by adding the emulsion to methanol without DPPH radical solution in the ratio of $1: 1$. All of the prepared samples were vortex-mixed and were kept in dark for an incubation period of $30 \mathrm{~min}$, followed by the detection of absorbance at $517 \mathrm{~nm}$ using a UV-vis spectrophotometer (Shanghai Precise Instrument Co., Ltd., Shanghai, China). Sampling was done after vortex mixing to assure reproducibility. The antioxidant activity of sea buckthorn oil-inwater emulsion was assessed by the percentage of DPPH that was decreased in comparison with that of the control condition after $30 \mathrm{~min}$ incubation. The following equation was used to calculate the DPPH radical scavenging activity for each sample:

$\mathrm{DPPH}$ radical scavenging activity $(\%)$

$$
=\left(1-\frac{A_{\text {sample }}-A_{\text {blank }}}{A_{\text {control }}}\right) \times 100 \% \text {, }
$$

where $A_{\text {sample }}, A_{\text {blank }}$, and $A_{\text {control }}$ were the absorbance of sample, blank, and control solutions, respectively.

2.6. Peroxide Value of the Emulsions. Peroxide value (PV) of each sample was quantitated applying the method reported by Shanta and Decker (1994). $1 \mathrm{~mL}$ of the emulsion was added to $5 \mathrm{~mL}$ of isooctane-isopropanol mixture $(2: 1 ; \mathrm{v} / \mathrm{v})$, followed by vortexing for $30 \mathrm{~s}$. After centrifugation for $2 \mathrm{~min}$ at $3000 \mathrm{rpm}$, the clear upper layer (sample extract) was collected. For the determination, $20 \mathrm{~mL}$ of potassium thiocyanate solution was mixed with the sample extract $(2 \mathrm{~mL})$, and methanol/1-butanol $(2: 1 ; \mathrm{v} / \mathrm{v})$ solution was added to $5 \mathrm{~mL}$ and then vortexed. The mixture was left for $20 \mathrm{~min}$ at dark, and the absorbance at $510 \mathrm{~nm}$ was determined $\left(A_{\text {lipid }}\right)$, using methanol/1-butanol $(2: 1 ; \mathrm{v} / \mathrm{v})$ solution as the reference. Second, another $2 \mathrm{~mL}$ of the sample extract was mixed with $20 \mu \mathrm{L}$ potassium thiocyanate and $20 \mu \mathrm{L}$ ferrous chloride solutions, and methanol/1-butanol (2:1; v/ v) solution was added to $5 \mathrm{~mL}$. After the same incubation procedure, the absorbance at $510 \mathrm{~nm}$ was determined ( $\left.A_{\text {sample }}\right)$. Third, $2 \mathrm{~mL}$ of distilled water was mixed with potassium thiocyanate solution, and methanol/1-butanol (2: $1 ; \mathrm{v} / \mathrm{v})$ solution was added to $5 \mathrm{~mL}$. After the same incubation procedure, the absorbance at $510 \mathrm{~nm}$ was determined
$\left(A_{\text {water }}\right)$. PV of the samples was determined using the following equation:

$$
\mathrm{PV}(\mathrm{meq} / \mathrm{kg})=\frac{A \times K \times n \times 0.5}{55.86 \times m \times 2} \times 1000,
$$

where $A=A_{\text {sample }}-\left(A_{\text {water }}+A_{\text {lipid }}\right), K$ is the slope of $\mathrm{Fe}^{3+}$ standard curve (1.5238), $m$ is the mass of the oil in the sample, and $n$ is the volume fraction of the sample extract.

2.7. Statistical Analysis. Statistical analysis was performed using OriginPro 7.5. All of the measurements were repeated three times. A one-way analysis of variance (ANOVA), followed by Tukey's test, was applied to determine significant differences between the mean values of each set of replicates. A significance level of $p<0.05$ was used throughout the study.

\section{Results and Discussion}

3.1. Physical Properties of Sea Buckthorn Oil-in-Water Emulsions. Emulsions with smaller particle size are generally produced by applying higher homogenization pressure. During the homogenization process, a premixed dispersion (also called coarse emulsion) of oil and water phases is forced to pass homogenizing valves and experienced collision, cavitation, shearing, friction, and some other forces to disrupt the oil phase into small droplets. In the meantime, amphiphilic ingredients are adsorbed onto the droplet surface, creating a stabilizing interfacial layer, which prevents the favorable aggregation of the newly produced droplets and finally leads to a fine emulsion [8]. Table 1 shows that droplet size of sea buckthorn oil emulsions (SC $1 \% \mathrm{~g} / 100 \mathrm{~g}$ ) decreased significantly with the increase in homogenization pressure, and the emulsions also had narrower particle size distribution (smaller PdI) at higher pressure. For example, the emulsion had a mean particle size of $282 \mathrm{~nm}$ and a PdI of 0.21 at $20 \mathrm{MPa}$, and particle size and PdI were decreased to $204 \mathrm{~nm}$ and 0.14 at $80 \mathrm{MPa}$. With sodium caseinate as the emulsifier, all the emulsions had high interfacial charges (|zeta-potential $\mid>30 \mathrm{mV}$ ), and the changes in homogenization pressure did not result in significant difference in zeta potential of the emulsions. To avoid droplet aggregation during homogenization, a sufficient interfacial coverage of emulsifier is essential [22]. When the oil droplets were not fully covered, emulsions usually had bigger particle size. On the contrary, selection of right emulsifiers would also help to stabilize the newly produced oil droplets. Table 2 presents that emulsions had increased particle size with higher oil content. It was due to the shortage of emulsifier at the interface of emulsions with higher oil content. Second, higher oil content contributed to emulsions with higher viscosity (data not shown), and the homogenization efficacy could be reduced, which can also lead to increased droplet size of the emulsions [23].

In the current study, roles of different emulsifiers on droplet size of sea buckthorn oil emulsions were also explored. Table 2 indicates that sugar ester was effective in forming emulsions with smaller particle size, and the mean 
TABLE 1: Droplet characteristics of the sea buckthorn oil-in-water emulsions (oil: $5 \mathrm{~g} / 100 \mathrm{~g}$; SC: $1 \mathrm{~g} / 100 \mathrm{~g}$ ) produced at different homogenization pressures.

\begin{tabular}{lcccc}
\hline $\begin{array}{l}\text { Pressure } \\
(\mathrm{MPa})\end{array}$ & 20 & 40 & 60 & 80 \\
\hline$d(\mathrm{~nm})$ & $282.1 \pm 2.3 \mathrm{a}$ & $231.3 \pm 4.8 \mathrm{~b}$ & $214.5 \pm 0.6 \mathrm{c}$ & $204.6 \pm 2.4 \mathrm{~d}$ \\
PdI & $0.21 \pm 0.02$ & $0.20 \pm 0.01$ & $0.17 \pm 0.02$ & $0.14 \pm 0.03$ \\
Zeta $(\mathrm{mV})$ & $-31.2 \pm 0.8 \mathrm{a}$ & $-32.6 \pm 0.4 \mathrm{a}$ & $-32.1 \pm 0.4 \mathrm{a}$ & $-30.6 \pm 0.5 \mathrm{a}$ \\
\hline
\end{tabular}

$\mathrm{d}$ : mean diameter and PdI: polydispersity index. Different letters in each row indicate significant differences among samples $(p<0.05)$.

TABle 2: Droplet size ( $\mathrm{nm}$ ) of the emulsions stabilized by sodium caseinate (SC), soy protein isolate (SPI), and sugar ester (SE) with different oil contents homogenized at $20 \mathrm{MPa}$.

\begin{tabular}{lccc}
\hline $\begin{array}{l}\text { Oil content } \\
(\mathrm{g} / 100 \mathrm{~g})\end{array}$ & SC & SPI & SE \\
\hline 5 & $282.1 \pm 2.3 \mathrm{aA}$ & $463.3 \pm 1.4 \mathrm{bA}$ & $278.6 \pm 2.9 \mathrm{aA}$ \\
10 & $301.5 \pm 2.0 \mathrm{aB}$ & $769.7 \pm 11.5 \mathrm{bB}$ & $299.1 \pm 2.2 \mathrm{aB}$ \\
20 & $355.3 \pm 2.8 \mathrm{aC}$ & $931.0 \pm 22.1 \mathrm{bC}$ & $354.2 \pm 2.8 \mathrm{aC}$ \\
\hline
\end{tabular}

Different lowercase letters in each row indicate significant differences among samples of different emulsifiers, and different uppercase letters in each row indicate significant differences among samples of different oil contents $(p<0.05)$.

droplet size of the emulsions stabilized by sodium caseinate and sugar ester had no significant difference regardless of oil content. Emulsions with sugar esters mostly had smaller PdI (Table 3), which could be attributed to the relatively smaller molecular weight of sugar ester, and it was able to quickly get adsorbed onto the droplet surface and reduce surface tension. The emulsions stabilized by the soy protein isolate mostly had bigger droplet size and wider size distribution (Tables 2 and 3). Moreover, oil content had a more significant effect on the droplet size of SPI-stabilized emulsions than SC- or SE-stabilized ones. For example, the SPI emulsion had a droplet size of $463 \mathrm{~nm}$ with $5 \mathrm{~g} / 100 \mathrm{~g}$ of the oil phase, and the size increased dramatically to 769.7 with $10 \mathrm{~g} / 100 \mathrm{~g}$ of the oil phase, while droplet size of SE emulsions was $278.6 \mathrm{~nm}$ with $5 \mathrm{~g} / 100 \mathrm{~g}$ of the oil phase and $299.1 \mathrm{~nm}$ with $10 \mathrm{~g} / 100 \mathrm{~g}$ of the oil phase, respectively. The relatively poor emulsifying property of SPI was the result of its closepacked globular and larger molecular size conformation, which inhibited its diffusion at the interface. Although SC also has large molecular weight, its conformation is more flexible and can be modified to facilitate its adsorption at the interface [24]. It should be pointed out the PDI values higher than 0.3 were just for indication purposes, as the emulsions had multimodal distribution, and they could contain too big droplets, which might be beyond the limit of the machine.

Emulsions are thermodynamically unstable due to the exceeded free energy of mixing from the large interfacial area of the aqueous and oil phases. An unstable emulsion can behave as flocculation, coalescence, creaming or sedimentation, Ostwald ripening, or phase inversion. In most situations, two or more types of the instability coexist or take place sequentially [8]. Stability test was carried out on SCstabilized emulsions with different oil contents by
TABle 3: Polydispersity index (PdI) of the emulsions stabilized by sodium caseinate (SC), soy protein isolate (SPI), and sugar ester (SE) with different oil contents homogenized at $20 \mathrm{MPa}$.

\begin{tabular}{lccc}
\hline Oil content $(\mathrm{g} / 100 \mathrm{~g})$ & SC & SPI & SE \\
\hline 5 & $0.21 \pm 0.02$ & $0.19 \pm 0.01$ & $0.18 \pm 0.03$ \\
10 & $0.16 \pm 0.02$ & $0.37 \pm 0.04$ & $0.15 \pm 0.03$ \\
20 & $0.17 \pm 0.03$ & $0.87 \pm 0.22$ & $0.19 \pm 0.02$ \\
\hline
\end{tabular}

centrifuging samples with LUMiSizer. Figure 1 shows the evolution of transmitted light during centrifuging of the emulsions with different oil contents. The increase in the transmission intensity of the lower part of the sample cuvettes (right side of the $X$-axis) suggested that the sample became clearer due to the lowered concentration of the oil droplets as they were moving to the upper part of the cuvettes (left side of the $X$-axis) because of droplet aggregation and density difference in the oil phase and water phase. When sufficient oil droplets were moved to the top of the emulsions, creaming could be observed. Among the three emulsions tested, the emulsion with $20 \mathrm{~g} / 100 \mathrm{~g}$ of the oil phase had the biggest change in light intensity (from 5\% at the beginning to $\sim 95 \%$ at the end), and the most part of the sample cuvettes experienced transmission change (from 98 to $114 \mathrm{~mm}$ ), indicating that this emulsion had the worst stability. The result was in agreement with particle size measurement of the emulsion during storage for $6 \mathrm{~d}$ (from 355 to $533 \mathrm{~nm}$ ). The instability was due to the shortage of emulsifier to cover the oil droplets in emulsions with higher oil content, and droplet aggregation through bridging took place during storage [20]. It was worth pointing out that the current study was an accelerated stability test as it was carried out within a centrifuge field. In fact, all the emulsions presented good stability without visible creaming during a storage test at room temperature for $30 \mathrm{~d}$.

3.2. Antioxidant Activity of Sea Buckthorn Oil-in-Water Emulsions. Sea buckthorn oils are rich in antioxidant compounds, e.g., vitamin A\&E, sterols, and flavonoids [25]. Effects of droplet size, oil content, and emulsifier type on the overall antioxidant activity of sea buckthorn oil emulsions were explored. Figure 2 presents the DPPH radical scavenging activity of the emulsions with different droplet sizes as influenced by homogenization pressures. The emulsion processed at $20 \mathrm{MPa}$ (particle size $282 \mathrm{~nm}$ ) showed the highest radical scavenging activity, and the emulsions processed at 40 (particle size $231 \mathrm{~nm}$ ), 60 (particle size $214 \mathrm{~nm}$ ), and $80 \mathrm{MPa}$ (particle size $204 \mathrm{~nm}$ ) presented no significant difference in radical scavenging activity. During homogenization at higher pressure, a temperature rise in the emulsions was widely observed, which may have caused degradation of some antioxidant compounds in the emulsions, leading to reduced antioxidant activity [26]. Second, the lipophilic antioxidants in the sea buckthorn oil may have higher local concentration in bigger droplets than smaller droplets, and they were also more distributed at the relatively smaller interface of the bigger particles. Third, the homogenization process can trigger the formation of free 


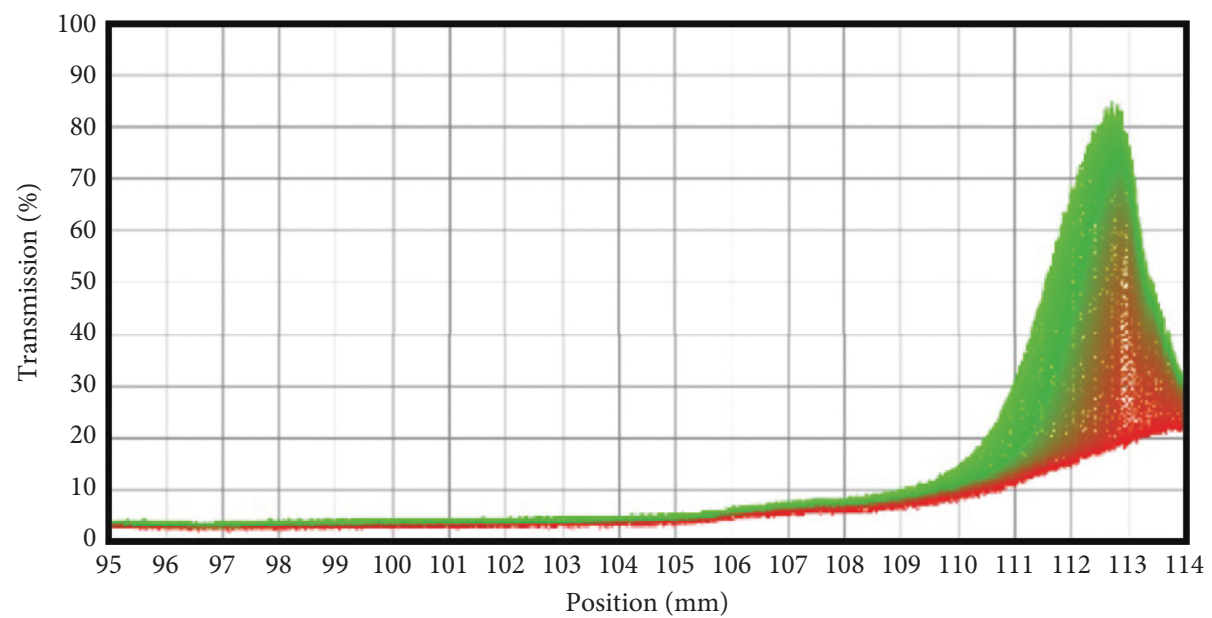

(a)

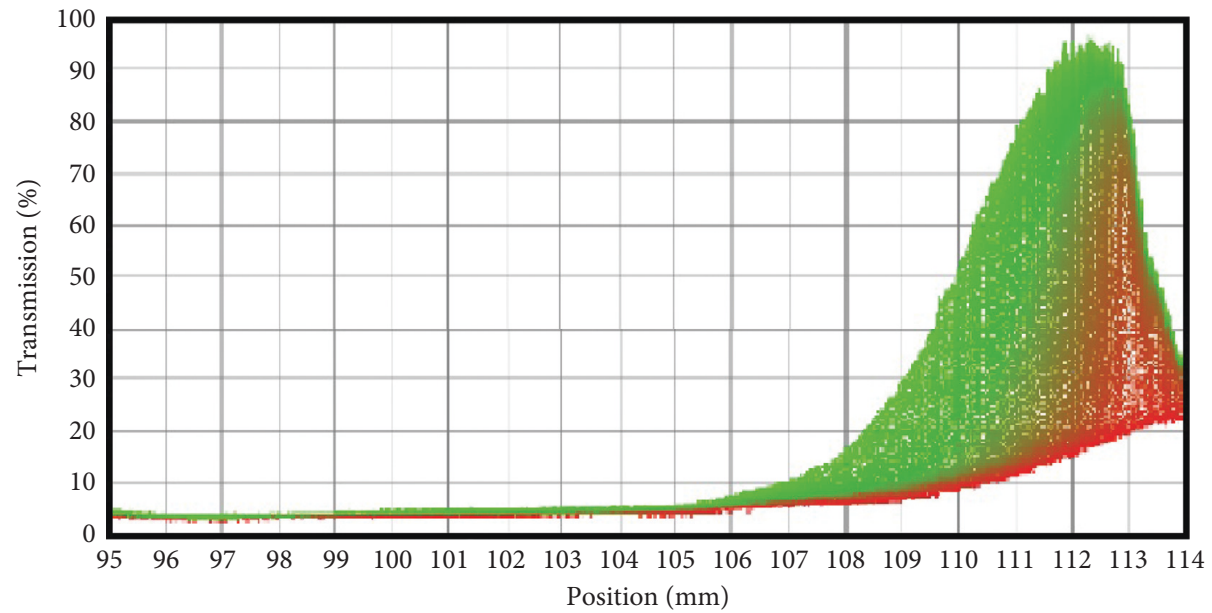

(b)

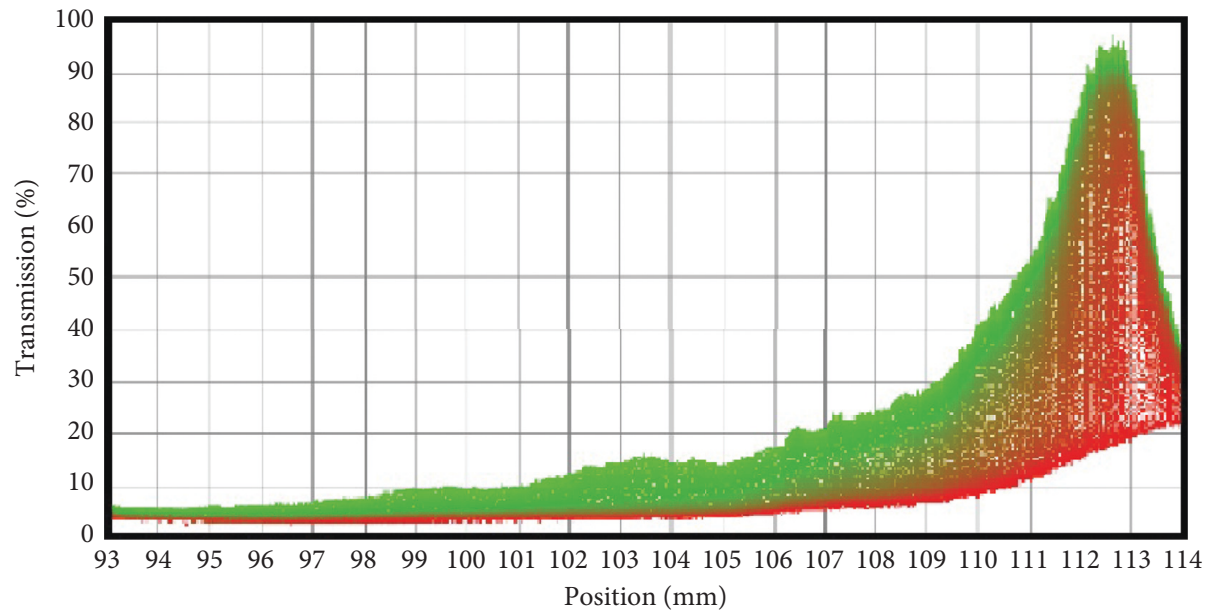

(c)

Figure 1: Evolution of transmission light signals of emulsions with different oil contents: (a) $5 \mathrm{~g} / 100 \mathrm{~g}$; (b) $10 \mathrm{~g} / 100 \mathrm{~g}$; (c) $20 \mathrm{~g} / 100 \mathrm{~g}$. The bottom red line represents the first scanning profile, and the top green line represents the last scanning profile.

radicals, with a higher intensity of cavitation and shearing, increasing the rate of free-radical formation [27]. The free radical formed could react with the antioxidants in the emulsions, and thus, fewer antioxidants were available to scavenge DPPH radicals. Many previous studies suggest that emulsions with smaller particles could accelerate the oxidation of the oil phase and reduce the bioactives within the oil droplets [27, 28]. Therefore, lower homogenization 


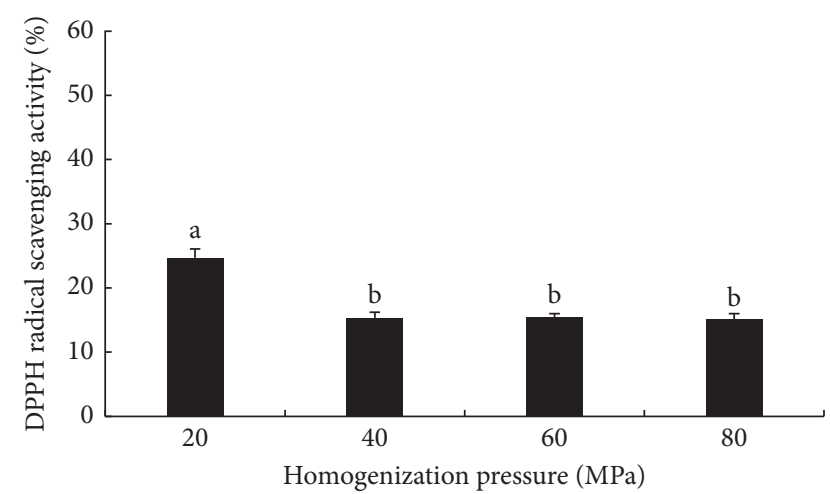

FIGURE 2: DPPH radical scavenging activity of the emulsions (oil: $5 \mathrm{~g} / 100 \mathrm{~g}$; SC: $1 \mathrm{~g} / 100 \mathrm{~g}$ ) processed at different pressures. Different letters above bars indicate significant differences among samples $(p<0.05)$.

pressure might be beneficial to retain the antioxidant activity of the sea buckthorn oil in emulsions. However, it was still not clear about the mechanism for the unchanged antioxidant activity of the emulsions processed at 40,60, and $80 \mathrm{MPa}$.

Figure 3 demonstrates that DPPH radical scavenging activity increased with oil content due to the increased amount of antioxidants in the oil phase of the emulsions. Moreover, the relatively bigger droplet size of the high oilloading emulsions helped to maintain the antioxidant activity of the emulsions. The scavenging activity was not increased linearly with oil content, which revealed that the concentration of antioxidants in the oil phase was not the only factor determining the overall antioxidant activity of the emulsions. Other factors, including droplet size, interface structure and composition, and emulsion stability are also important. When different emulsifiers were used to stabilize the emulsions, the emulsion containing SPI presented the highest radical scavenging activity, followed by the emulsions containing sodium caseinate and sugar ester. The underlying mechanism has been well documented in literatures as proteins contain antioxidant amino acids (e.g., lysine, methionine, and phenylalanine) in their molecular structures [24]. As SPI also contains cysteine, which has strong antioxidant activity, the SPI-stabilized sea buckthorn oil emulsion was effective in scavenging DPPH radicals. Additionally, the bigger size of the SPI emulsion also worked to improve its antioxidant activity. However, this finding was contradictory to the study by $\mathrm{Hu}$ et al. [24], who reported that the emulsion stabilized by casein had lower lipid oxidation than the emulsion stabilized by SPI. The authors acknowledged the roles of antioxidant amino acids, but they attributed the stronger antioxidant activity of casein-stabilized emulsion to the thicker interface formed by casein. However, SPI was also able to form a thicker interface around oil droplets. The contradiction in the two studies could be due to the large differences in emulsion composition and production procedures. In the current study, the oil phase was a mixture of different compounds, and they may have interactions with the emulsifiers, which could then affect the overall antioxidant activity of the system.

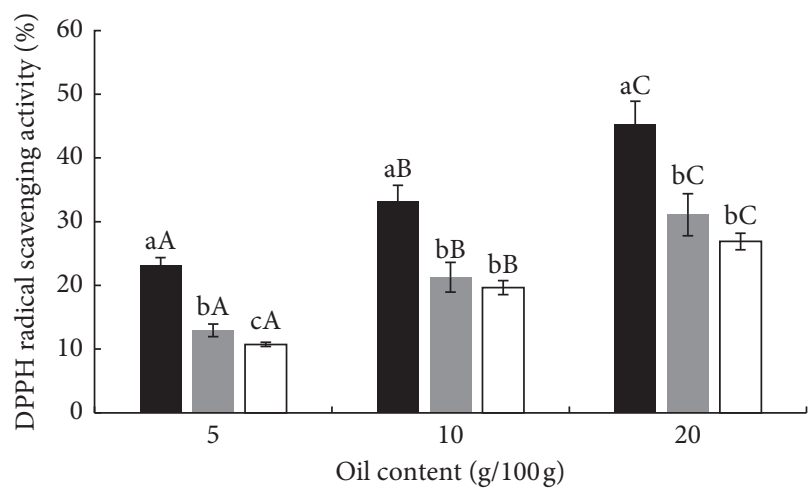

Figure 3: DPPH radical scavenging activity of the emulsions with different oil contents and emulsifiers ( $\square$ SPI, $\square$ SC, and $\square$ SE). Different lowercase letters above bars indicate significant differences among samples of different emulsifiers, and different uppercase letters above bars indicate significant differences among samples of different oil contents $(p<0.05)$.

As discussed previously, emulsions were unstable during storage, and droplet aggregation, creaming, and phase separation could affect the chemical stability of the oil phase. Figure 4 shows the changes in DPPH radical scavenging activity of the emulsions with different oil contents during storage at room temperature. All the emulsions had decreased antioxidant activity along the time scale, which was the result of the degradation of the antioxidant compounds in the oil phase. Vitamins, flavonoids, sterols, and unsaturated fatty acids were sensitive to light and oxygen. Oils were initially much more stable to oxidation in bulk systems than in the corresponding oil-in-water emulsions as the large interface covering oil droplets allowed more contact of the oil with the environment stresses, particularly irons and oxygen brought in during emulsion preparation [29]. Therefore, the antioxidant activity of the emulsions may decrease faster than that of pure oils. However, many studies also reported that, by a careful selection of biopolymers, a compact interfacial film could be formed around oil droplets, which would allow higher stability of the oil phase and improved antioxidant activity of the emulsion systems [30]. Oil content did not show big effect on the decreased antioxidant activity of the emulsions. After $3 \mathrm{~d}$ of storage, about $\sim 88 \%$ of the original DPPH radical scavenging activity was retained in each emulsion. After $6 \mathrm{~d}$ of storage, about $\sim 67 \%$ of the original antioxidant activity was retained in the systems containing 5 and $10 \mathrm{~g} / 100 \mathrm{~g}$ of the oil phase and about $76 \%$ in the system containing $20 \mathrm{~g} / 100 \mathrm{~g}$ of the oil phase. The relatively higher antioxidant stability of high oilloading system could be the result of its bigger droplet size. To better understand the oxidation stability of the emulsions, the peroxide values (PVs) were also determined (Figure 5). The results supported the conclusion made in antioxidant stability analysis (Figure 4). With the increase of storage time, higher PVs were observed in all the emulsions due to the oxidation of the oil phase. Comparatively, the emulsion containing 5\% oil had the fastest increase in PV due to the lowest content of antioxidant ingredients in the oil and the smallest particle size of the oil phase. Therefore, the lower 


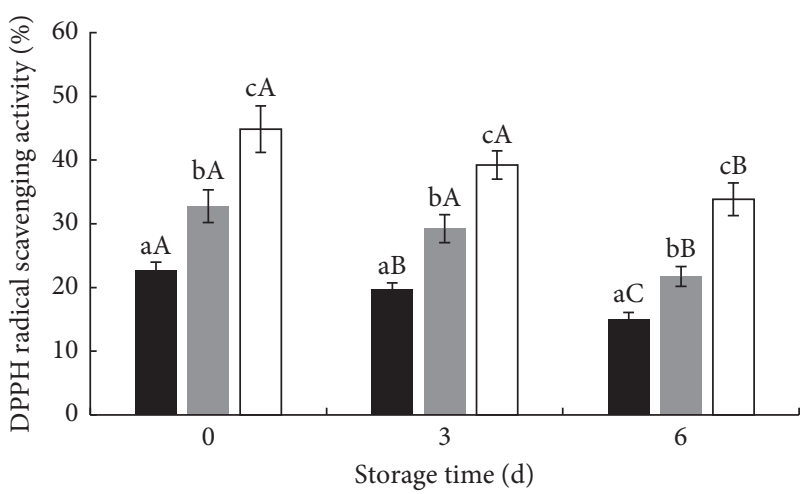

FIGURE 4: Changes in DPPH radical scavenging activity in a 6-day storage test of the emulsions with different oil contents ( $\square$ oil: $5 \mathrm{~g}$ / $100 \mathrm{~g}$; oil: $10 \mathrm{~g} / 100 \mathrm{~g}$; $\square$ oil: $20 \mathrm{~g} / 100 \mathrm{~g}$ ). Different lowercase letters above bars indicate significant differences among samples of different oil contents, and different uppercase letters above bars indicate significant differences among samples of different storage times $(p<0.05)$.

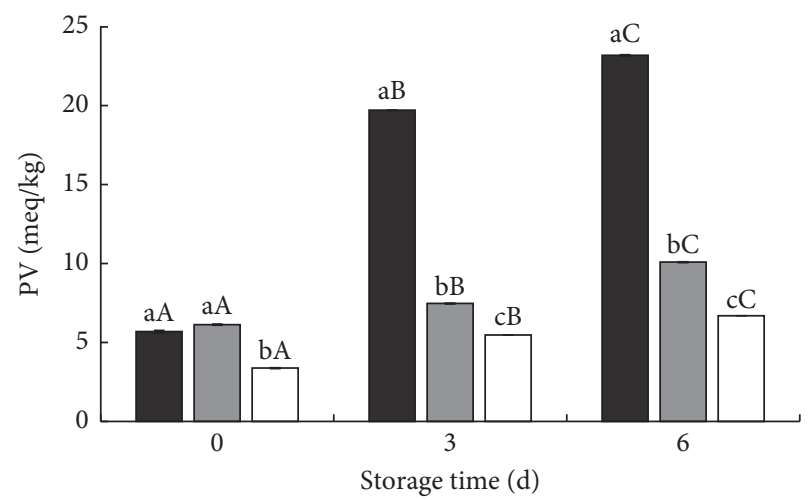

FIGURe 5: Peroxide values (PVs) of the emulsions with different oil contents ( $\square$ oil: 5 g/100 g; $\square$ oil: 10 g/100 g; $\square$ oil: 20 g/100 g) during storage. Different lowercase letters above bars indicate significant differences among samples of different oil contents, and different uppercase letters above bars indicate significant differences among samples of different storage times $(p<0.05)$.

chemical stability of the emulsions contributed to lower $\mathrm{DPPH}$ radical scavenging activity of the systems.

\section{Conclusions}

The current study mainly investigated the effect of emulsifier type and oil content on the antioxidant properties of sea buckthorn oil-in-water emulsions, aimed to facilitate wider applications of sea buckthorn oil in food. The overall antioxidant activity of the emulsions expressed as their DPPH radical scavenging activity was dependent on properties and compositions of the emulsions. The study showed that higher radical scavenging activity could be obtained in emulsions with bigger droplets, higher oil content, or using soy protein isolate as the emulsifier. However, the bioactives in the emulsions could experience degradation during storage, which then led to lower antioxidant activity. As complex systems, sea buckthorn oil-in-water emulsions showed different antioxidant characteristics from the bulk oil, and it seemed that the overall antioxidant activity of the emulsions could be modulated by adjusting properties and compositions of the emulsions.

\section{Data Availability}

The data used to support the findings of this study are available from the corresponding author upon request.

\section{Conflicts of Interest}

The authors declare that there are no conflicts of interest regarding the publication of this article.

\section{Acknowledgments}

This research was funded by the National Natural Science Foundation of China (no. 31701648).

\section{References}

[1] T. S. C. Li and W. R. Schroeder, "Sea buckthorn (Hippophae rhamnoides L.): a multipurpose plant," HortTechnology, vol. 6, no. 4, pp. 370-380, 1996.

[2] B. Olas, "The beneficial health aspects of sea buckthorn (Elaeagnus rhamnoides (L.) A. Nelson) oil," Journal of Ethnopharmacology, vol. 213, pp. 183-190, 2018.

[3] B. Olas, "Sea buckthorn as a source of important bioactive compounds in cardiovascular diseases," Food and Chemical Toxicology, vol. 97, pp. 199-204, 2016.

[4] G. Suryakumar and A. Gupta, "Medicinal and therapeutic potential of Sea buckthorn (Hippophae rhamnoides L.)," Journal of Ethnopharmacology, vol. 138, no. 2, pp. 268-278, 2011.

[5] L. M. Bal, V. Meda, S. N. Naik, and S. Satya, "Sea buckthorn berries: a potential source of valuable nutrients for nutraceuticals and cosmoceuticals," Food Research International, vol. 44, no. 7, pp. 1718-1727, 2011.

[6] L. Mao and S. Miao, "Structuring food emulsions to improve nutrient delivery during digestion," Food Engineering Reviews, vol. 7, no. 4, pp. 439-451, 2015.

[7] D. J. McClements, "Emulsion design to improve the delivery of functional lipophilic components," Annual Review of Food Science and Technology, vol. 1, no. 1, pp. 241-269, 2010.

[8] D. J. McClements, Food Emulsions: Principles, Practices and Techniques, CRC Press, Boca Raton, FL, USA, 3rd edition, 2015.

[9] L. Mao, D. Wang, F. Liu, and Y. Gao, "Emulsion design for the delivery of $\beta$-carotene in complex food systems," Critical Reviews in Food Science and Nutrition, vol. 58, no. 5, pp. 770-784, 2018.

[10] D. J. McClements and S. M. Jafari, "Improving emulsion formation, stability and performance using mixed emulsifiers: a review," Advances in Colloid and Interface Science, vol. 251, pp. 55-79, 2018.

[11] H. Y. Song, T. W. Moon, and S. J. Choi, "Impact of antioxidant on the stability of $\beta$-carotene in model beverage emulsions: role of emulsion interfacial membrane," Food Chemistry, vol. 279, pp. 194-201, 2019.

[12] W. L. Porter, "Paradoxical behavior of antioxidants in food and biological systems," Toxicology and Industrial Health, vol. 9, no. 1-2, pp. 93-122, 1993. 
[13] F. Shahidi and Y. Zhong, "Revisiting the polar paradox theory: a critical overview," Journal of Agricultural and Food Chemistry, vol. 59, no. 8, pp. 3499-3504, 2011.

[14] H. L. Tan and K. M. McGrath, "How does oil type determine emulsion characteristics in concentrated Na-caseinate emulsions?," Journal of Colloid and Interface Science, vol. 403, pp. 7-15, 2013.

[15] C. Qian, E. A. Decker, H. Xiao, and D. J. McClements, "Nanoemulsion delivery systems: influence of carrier oil on $\beta$-carotene bioaccessibility," Food Chemistry, vol. 135, no. 3, pp. 1440-1447, 2012.

[16] R. Partanen, P. Hakala, O. Sjövall, H. Kallio, and P. Forssell, "Effect of relative humidity on the oxidative stability of microencapsulated sea buckthorn seed oil," Journal of Food Science, vol. 70, no. 1, pp. E37-E43, 2005.

[17] L. Mihalcea, M. Turturică, I. O. Ghinea et al., "Encapsulation of carotenoids from sea buckthorn extracted by $\mathrm{CO}_{2}$ supercritical fluids method within whey proteins isolates matrices," Innovative Food Science \& Emerging Technologies, vol. 42, pp. 120-129, 2017.

[18] R. V. Tonon, C. R. F. Grosso, and M. D. Hubinger, "Influence of emulsion composition and inlet air temperature on the microencapsulation of flaxseed oil by spray drying," Food Research International, vol. 44, no. 1, pp. 282-289, 2011.

[19] S. Cupara, S. Jankovic, I. Arsic et al., "Characterization of sea buckthorn oil emulsion," Military Medical Science Letters, vol. 81, pp. 56-60, 2011.

[20] L. Mao, Y. H. Roos, and S. Miao, "Effect of maltodextrins on the stability and release of volatile compounds of oil-in-water emulsions subjected to freeze-thaw treatment," Food $\mathrm{Hy}$ drocolloids, vol. 50, pp. 19-227, 2015.

[21] M. Yang, Y. Wu, J. Li, H. Zhou, and X. Wang, "Binding of curcumin with bovine serum albumin in the presence of I-carrageenan and implications on the stability and antioxidant activity of curcumin," Journal of Agricultural and Food Chemistry, vol. 61, no. 29, pp. 7150-7155, 2013.

[22] Z. Li, L. Dai, D. Wang, L. Mao, and Y. Gao, "Stabilization and rheology of concentrated emulsions using the natural emulsifiers quillaja saponins and rhamnolipids," Journal of Agricultural and Food Chemistry, vol. 66, no. 15, pp. 39223929, 2018.

[23] S. R. Derkach, "Rheology of emulsions," Advances in Colloid and Interface Science, vol. 151, no. 1-2, pp. 1-23, 2009.

[24] M. Hu, D. J. McClements, and E. A. Decker, "Lipid oxidation in corn oil-in-water emulsions stabilized by casein, whey protein isolate, and soy protein isolate," Journal of Agricultural and Food Chemistry, vol. 51, no. 6, pp. 1696-1700, 2003.

[25] F.-M. Ursache, I. O. Ghinea, M. Turturică, I. Aprodu, G. Râpeanu, and N. Stănciuc, "Phytochemicals content and antioxidant properties of sea buckthorn (Hippophae rhamnoides L.) as affected by heat treatment-quantitative spectroscopic and kinetic approaches," Food Chemistry, vol. 233, pp. 442-449, 2017.

[26] L. Mao, J. Yang, D. Xu, F. Yuan, and Y. Gao, "Effects of homogenization models and emulsifiers on the physicochemical properties of $\beta$-carotene nanoemulsions," Journal of Dispersion Science and Technology, vol. 31, no. 7, pp. 986-993, 2010.

[27] C. Tan and M. Nakajima, " $\beta$-Carotene nanodispersions: preparation, characterization and stability evaluation," Food Chemistry, vol. 92, no. 4, pp. 661-671, 2005.

[28] J. Yi, Y. Li, F. Zhong, and W. Yokoyama, "The physicochemical stability and in vitro bioaccessibility of beta-carotene in oil-in-water sodium caseinate emulsions," Food Hydrocolloids, vol. 35, pp. 19-27, 2014.

[29] T. Waraho, D. J. McClements, and E. A. Decker, "Mechanisms of lipid oxidation in food dispersions," Trends in Food Science \& Technology, vol. 22, no. 1, pp. 3-13, 2011.

[30] J. Feng, H. Cai, H. Wang, C. Li, and S. Liu, "Improved oxidative stability of fish oil emulsion by grafted ovalbumincatechin conjugates," Food Chemistry, vol. 241, pp. 60-69, 2018. 


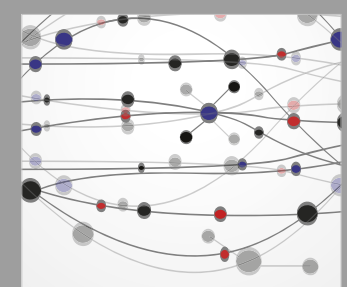

The Scientific World Journal
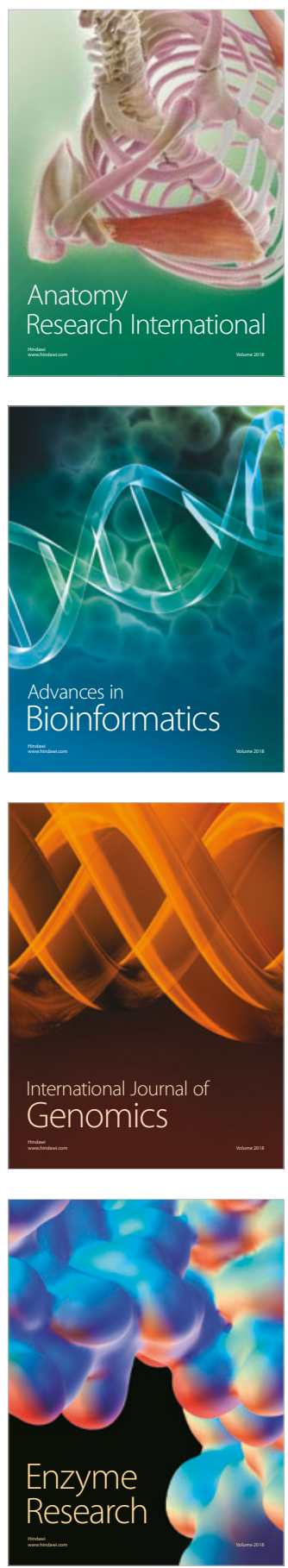
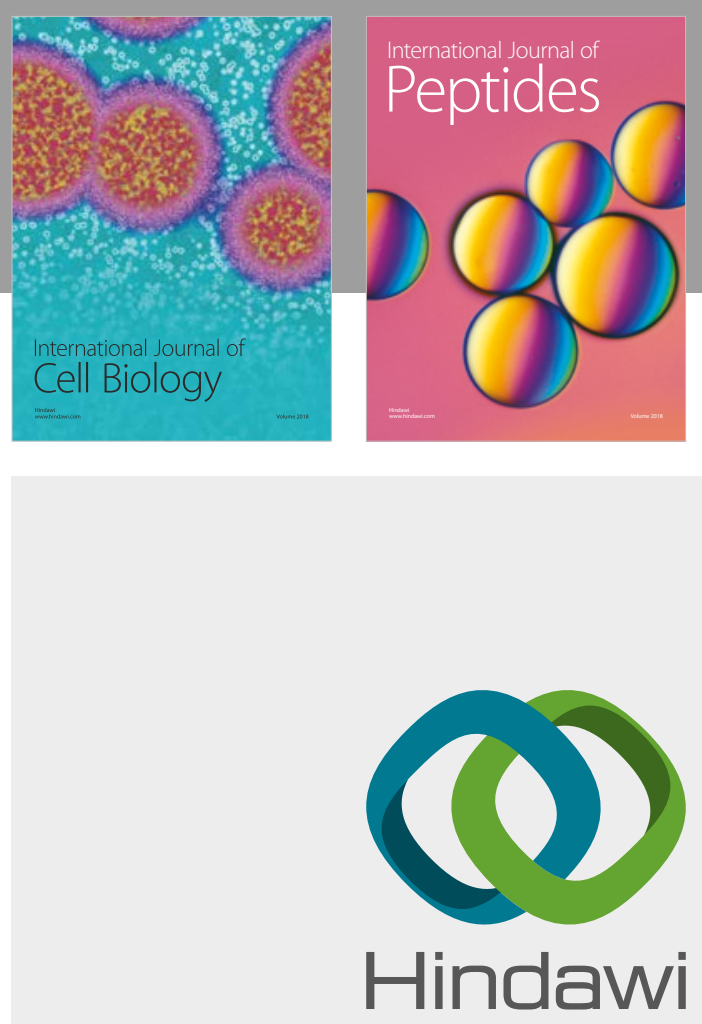

Submit your manuscripts at

www.hindawi.com
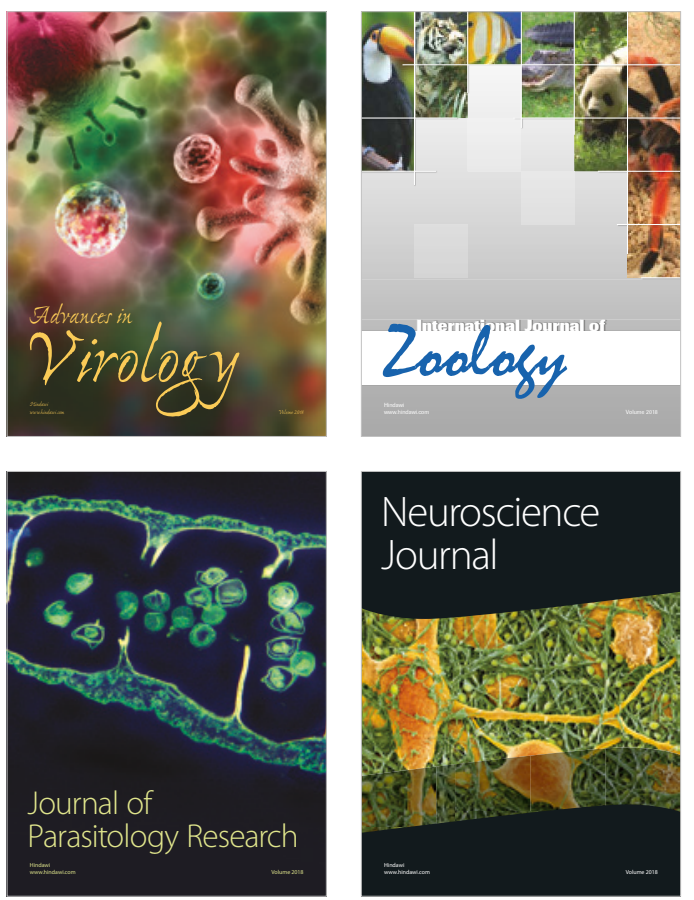
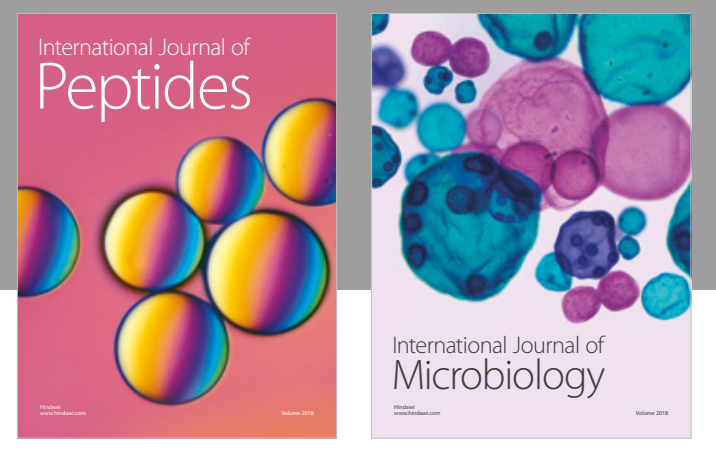

nternational Journal of Microbiology
Journal of
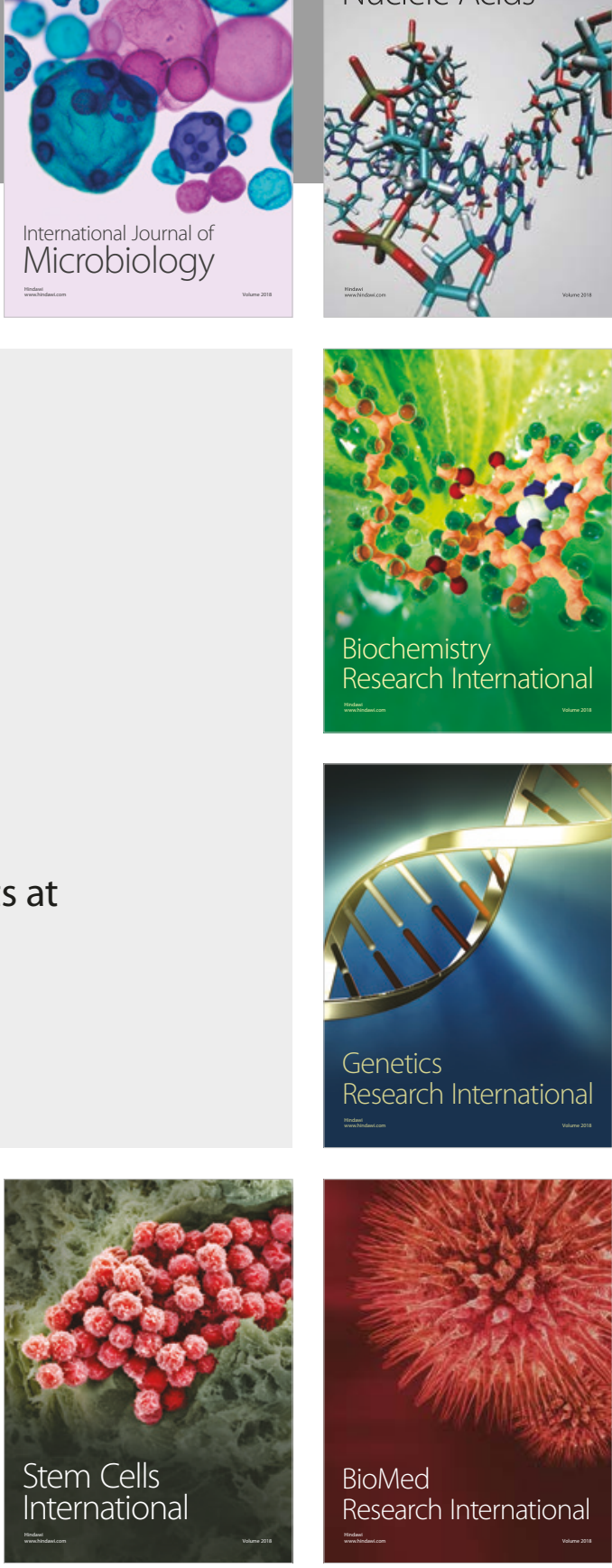
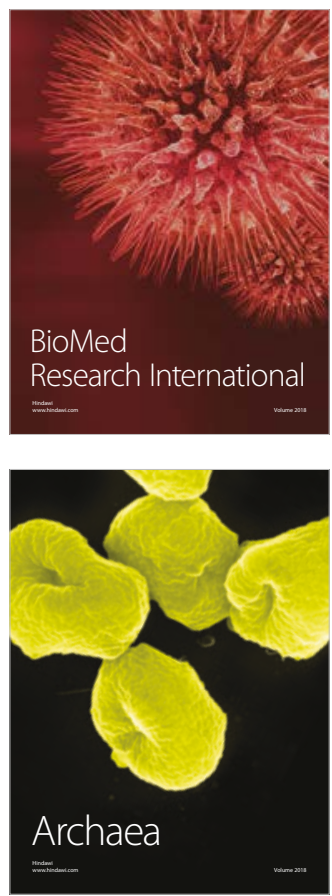